\title{
Expression of CASK Antibody in Non-Mucinous Colorectal Adenocarcinoma
}

\author{
SAHAR F. MANSOUR, M.D.; REHAM H ABDUL-AZIM, M.Sc.; NOHA R. NOUFAL, M.D. and \\ HOWAYDA ABD EL-AL, M.D.
}

The Department of Pathology, Faculty of Medicine, Suez Canal University

\begin{abstract}
Background: Colorectal cancers are one of the leading causes of cancer related morbidity and mortality worldwide. In Egypt was 4\% in the year 2012 ranking as the sixth cancer in Egypt and representing 53\% of GI malignancy. It is obvious that there is no single determining factor for the process of carcinogenesis or spread of colorectal carcinoma. There are many proposed contributing factors, including disturbances in Epithelial-Mesenchymal Transition (EMT) and loss of regulation of adhesion molecules and polarity proteins. Calcium/calmodulin-dependent serine protein kinase (CASK) belongs to the Membrane-Associated Guanylate Kinase (MAGUK) family. The role of CASK in Colorectal cancer hasn't been yet fully understood.
\end{abstract}

Aim of Study: To evaluate the expression of Calcium/calmodulin-dependent serine protein kinase (CASK) in non-mucinous colorectal adenocarcinoma and to evaluate its correlation with variable clinicopathological factors.

Material and Methods: It is retrospective descriptive study is conducted in the Department of Pathology, Faculty of Medicine, Suez Canal University Teaching Hospital. It include 42 archived paraffin blocks of non-mucinous colorectal adenocarcinoma of patients who underwent surgical excision without previous chemotherapy or radiation, 15 adenomatous polyps and 5 normal mucosa samples. The clinical and pathological data of studied patients are taken from medical records, pathology referral reports and pathology reports. Patients with incomplete data excluded from the study. Reviewing the data of survival from the archived registry files at department of Oncology, Suez Canal University Teaching Hospital. Sections cut into 5um thick sections and mounted on positive charged slides for immunohistochemical staining using CASK Antibody (S56A-50): Mouse Monoclonal, Novus Biologicals, Catalogue no. NBP1-47648. Staining intensity graded according to the Allred score on a 0-3 scale.

Data Analysis: The relation between CASK antibody staining intensity in the studied biopsies and other clinicopathological parameters including (age, gender, histopathological tumor features) was evaluated using Pearson's X2 test The level of statistical significance ( $p$-value) was set at $<0.05$. All analyses were performed using the statistical package for social science (IBM SPSS) program (Ver. 19).

Correspondence to: Dr. Sahar F. Mansour, The Department of Pathology, Faculty of Medicine, Suez Canal University
Results: The study revealed that CASK protein had mainly cytoplasmic and cytoplasmic-membranous pattern of expression. The CASK protein was overexpressed in the majority of CRC samples with $85.7 \%$ of cases showing moderate to strong expression, while only $14.3 \%$ of cases displayed minimal faint expression. On the other hand, CASK expression was low in the majority (53.3\%) of adenoma samples. Additionally, adenomas with high grade dysplasia showed stronger staining intensity for CASK protein and higher percentage of positive cases than the ones with low grade dysplasia by IHC. As regard to the association between CASK protein overexpression and clinicopathological prognostic factors we found that CASK demonstrated significantly higher expression in tumor samples with early stages (I/II) rather than advanced stage (III/IV). Low grade tumors showed higher percentage of positive cases and stronger intensity of staining for CASK protein than high grade tumors. Expression of CASK protein showed no statistically significant correlation with patients' gender or age respectively. There was also no significant correlation with tumor site, gross tumor size, histologic tumor border configuration, lymphovascular invasion or distant metastasis.

Conclusion: CASK protein was overexpressed in the majority of non-mucinous colorectal adenocarcinoma (CRC) cases with mainly cytoplasmic and cytoplasmic-membranous localization. However, its expression was significantly less in adenomas with a possible association with the grade of dysplasia; giving positive pattern in highly dysplastic cases suggesting a role in the progression of adenomas into carcinomas. CASK overexpression also associated with both TNM stage of tumors and their histologic grade. CASK was significantly overexpressed in early stage and low grade tumors rather than tumors with advanced stage and higher histological grades. This suggests that CASK protein is a good prognostic factor and might contribute in tumor confinement and localization.

Key Words: CASK - Cell-adhesion - Non-mucinous colorectal adenocarcinoma $(\mathrm{CRC})$ - Colonic adenoma.

\section{Introduction}

COLORECTAL cancers are one of the leading causes of cancer related morbidity and mortality worldwide and on a national level as well. It is the third most common cancer globally and the fourth 
leading cause of death [1]. In Egypt was 4\% in the year 2012 ranking as the sixth cancer in Egypt and representing 53\% of GI malignancy. Recent statistical data estimated the incidence of colorectal cancer among Egyptian males to $6.1 \%$ and slightly lower in females with a $5.2 \%$ incidence rate $[2,3]$ It is obvious that there is no single determining factor for the process of carcinogenesis or spread of colorectal carcinoma. There are many proposed contributing factors, including disturbances in Epithelial-Mesenchymal Transition (EMT) and loss of regulation of adhesion molecules and polarity proteins [4]

Calcium/calmodulin-dependent serine protein kinase (CASK) belongs to the Membrane-Associated Guanylate Kinase (MAGUK) family, which is characterized structurally by a tripartite domain structure: A Src homology 3 (SH3) domain, a domain with homology to the enzyme Guanylate Kinase (GUK), and a PDZ domain [5]. Its binding to syndecan-2 has been reported to mediate cellcell adhesion and proliferation, which can contribute to tumorogenesis. CASK has also been reported to bind the cytoskeletal adapter protein EPB41 (Protein 4.1) linking both the extracellular matrix and the intracellular cytoskeleton and thus contributing to the regulation of epithelial cell polarity. Loss of cell polarity is a defining criterion of malignant neoplasms [6]. The role of CASK in Colorectal cancer hasn't been yet fully understood. There is one study that linked CASK with the development and prognosis of colorectal cancer. This cannot be regarded as enough evidence to support or defy the fore mentioned hypothesis [7] Based on the above mentioned functions of CASK in regulating both cell-cell interaction and cell polarity, we hypothesized that genetic errors involving the CASK encoding gene (Reelin) might participate in tumorogenesis, cancer spread and metastasis. The role of CASK in Colorectal cancer hasn't been yet fully understood. There is one study that linked CASK with the development and prognosis of colorectal cancer. This cannot be regarded as enough evidence to support or defy the fore mentioned hypothesis.

In this study, we focused on studying the expression of CASK antibody in colorectal adenocarcinoma, its association with different prognostic factors mainly tumor stage, histological grade, vascular invasion and metastasis to evaluate its prognostic significance.

\section{Material and Methods}

It is retrospective descriptive study is conducted in the Department of Pathology, Faculty of Medi- cine, Suez Canal University Teaching Hospital. It include 42 archived paraffin blocks of nonmucinous colorectal adenocarcinoma of patients who underwent surgical excision without previous chemotherapy or radiation, 15 adenomatous polyps and 5 normal mucosa samples in period between January 2013 to January 2014. The clinical and pathological data including: Age, gender, site and size of the tumor, pathologic stage, grade, and metastasis will be taken from medical records, pathology referral reports and pathology reports. Patients with incomplete data will be excluded from the study. Reviewing the data of survival from the archived registry files at Department of Oncology, Suez Canal University Teaching Hospital.

Immunohistochemistry staining: Sections cut into 5 um thick sections and mounted on positive charged slides for immunohistochemical staining using CASK Antibody (S56A-50): Mouse Monoclonal, Novus Biologicals, Catalogue no. NBP147648. Staining intensity graded according to the Allred score on a 0-3 scale as follows: 0 (absence of staining), 1 (weakly stained), 2 (moderately stained), 3 (strongly stained). The percentage of positive tumor cells scored as follows: 0 (absence of positive cells), 1 ( $<33 \%$ positive tumor cells), 2 (33-66\% positive tumor cells), 3 (>66\% positive tumor cells). The staining score calculated as the staining intensity score $\mathrm{X}$ the percentage score; ranging from 0 to 9 (absence, staining score $=0$; weak, $0<$ staining score $\leq 4$; strong, $5 \leq$ staining score $\leq 9)$ using image analysis in counting staining score (Wei et al., 2014).

\section{Data analysis:}

The relation between CASK antibody staining intensity in the studied biopsies and other clinicopathological parameters including (age, gender, histopathological tumor features) was evaluated using Pearson's $\chi$ test. The level of statistical significance ( $p$-value) was set at $<0.05$. All analyses were performed using the statistical package for social science (IBM SPSS) program (Ver. 19).

\section{Results}

\section{Demographic criteria of study population:}

The study involved 42 cases of non-mucinous adenocarcinoma, 15 cases of colorectal adenomas. 42 cases of non-mucinous adenocarcinoma included $40.5 \%$ male patients and $59.5 \%$ females with mean age of \pm 45 years. 15 adenoma cases included $53.3 \%$ males and $46.7 \%$ female patients with mean age \pm 47 years. 


\section{Histopathological features:}

Histological subtypes of the adenomas group included tubulovillous subtype which represented the majority of cases $(80 \%)$, while $13.3 \%$ of cases were of the tubular subtype and only $6.7 \%$ of the villous subtype ( $\mathrm{SD} \pm 0.723)$. Cases with low grade dysplasia represented $53.3 \%$, while $46.7 \%$ of cases showed high grade dysplasia with a SD \pm 0.516 .

Out of the 42 CRC cases, $59.5 \%$ were of low grade while $40.5 \%$ of cases were of high grade with a Std. Dev. of \pm 0.497 . Percentage of cases at TNM stage III was $16.7 \%$ cases of CRC were at stage I, $19 \%$ cases were stage II, $47.6 \%$ cases were stage III and $16.7 \%$ cases were stage IV. None of the studied tumors were at stage T1, while the majority of cases were diagnosed at advanced tumor (T) stage; $66.7 \%$ of examined samples were at pT3 and pT4. Metastatic deposits in lymph nodes were evident in $69.1 \%$ of cases, while $16.7 \%$ cases demonstrated evidence of distant metastasis. Lymphovascular invasion was assessed in $38.1 \%$ of cases. Lymphovascular invasion was associated with advanced tumor stage.

\section{IHC expression of CASK protein:}

The expression of CASK protein was evaluated by immunohistochemical technique on both CRC and adenomas samples. Normal colonic mucosa at the periphery of examined tumor sections were used as internal control.

Immnuohistochemistry showed mainly cytoplasmic and cytomembranous pattern of expression for CASK protein, with focal localization at the basolateral borders of normal mucosa cells (internal control) which was given an IS of + 1 and compared the IS of malignant cells accordingly. The ganglionic cells of the myenteric plexus between muscularis propria layers were positive for CASK and were used as an internal control as well Fig. (1).

The CASK protein was overexpressed in the majority of CRC samples with $85.7 \%$ of cases showing moderate to strong expression, while only $14.3 \%$ of cases displayed minimal faint expression. On the other hand, CASK expression was low in the majority of adenoma samples; $53.3 \%$ of cases showed low expression while only $6.67 \%$ showed strong expression. Nearly half $(45.2 \%)$ of CRC samples showed both cytoplasmic and cytoplasmmembranous expression by immunohistochemistry Figs. (2-4), while only $20 \%$ of adenomas samples showed dual pattern of staining. Membranous staining pattern showed a statistically significant correlation with CASK protein overexpression ( $p$ value $<0.05$ ).
On the other hand, CASK expression was low in the majority of adenoma samples; $53.3 \%$ of cases showed low expression while only $6.67 \%$ showed strong expression. Pattern of staining showed no statistical significance in the adenomas group. No statistical significance was demonstrated between cytoplasmic staining pattern and either tumor TNM stage or grade ( $p$-value $>0.05$ ).

Nearly half (45.2\%) of CRC samples showed both cytoplasmic and cytomembranous expression by immunohistochemistry, while only $20 \%$ of adenomas samples showed dual pattern of staining. Membranous staining pattern showed a statistically significant correlation with CASK protein overexpression ( $p$-value <0.05). Pattern of staining showed no statistical significance in the adenomas group (Table 1).

Cytomembranous staining pattern was significantly correlated with CASK protein expression in colorectal cancer samples ( $p$-value $<0.05$ ). Membranous staining was the dominant pattern among low grade CRC samples Fig. (5) and showed significant correlation ( $p$-value $<0.05)$ with early stage tumors (I/II) as out of the $54.8 \%$ of cases with membranous pattern of staining for CASK protein, $28.6 \%$ were at early stages (I/II), while $38.1 \%$ were of advanced tumor stage (III/IV). Membranous staining pattern showed a statistically significant relation with the TNM stage ( $p$-value $<0.05$ ), with association with the early tumor stages. Tumor samples excised at an early stage mainly expressed CASK protein on their cytomembranes. Correlation between membranous expression of CASK protein and histologic grade of tumors revealed no statistical significance. Out of the $54.8 \%$ of cases with membranous expression of CASK, $38.1 \%$ were of low grade while $16.7 \%$ were of high grade.

\section{Association of CASK with clinicopathological features of CRC: \\ Expression of CASK protein showed no statis-} tically significant correlation with patients' gender or age respectively $(p>0.05)$. There was also no significant correlation with tumor site, gross tumor size, histologic tumor border configuration, lymphovascular invasion or distant metastasis. The correlation of CASK expression with clustered TNM stage (stages I/II vas III/IV) was statistically significant $(p<0.05)$, with significantly higher expression in tumor samples with early stages (I/II) rather than advanced stage (III/IV) (Table 2).

Low grade CRC samples that showed strong degree of CASK overexpression represented 33.3\% out of the total number of cases Figs. $(6,7)$, while 
only $4.8 \%$ of cases with high grade carcinoma showed strong staining intensity Fig. (8). The correlation of CASK expression and histologic grade of differentiation was statistically significant $(p<0.05)$ (Table 2$)$, with low grade tumors showing higher percentage of positive cases and stronger intensity of staining for CASK protein expression than high grade tumors.

\section{Association of CASK and dysplasia in adenomas:}

Out of the examined adenoma specimens 53.3\% showed low grade dysplasia Fig. (9), while 46.7\%

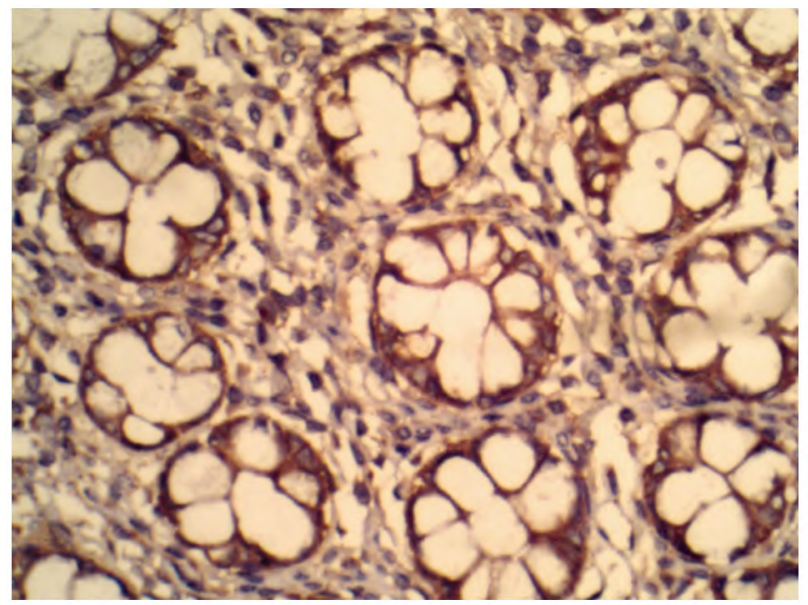

(A) were of high grade Fig. (10). We measured the association between CASK protein overexpression and the degree of dysplasia. There was a reasonable association, where highly dysplastic adenomas showing stronger staining intensity and higher percentage of positive cases (Table 3).

Adenoma samples with high grade dysplasia that overexpressed CASK protein represented $33.3 \%$ Fig. (11), while only $13.3 \%$ of cases demonstrated low grade dysplasia and CASK overexpression Fig. (12).

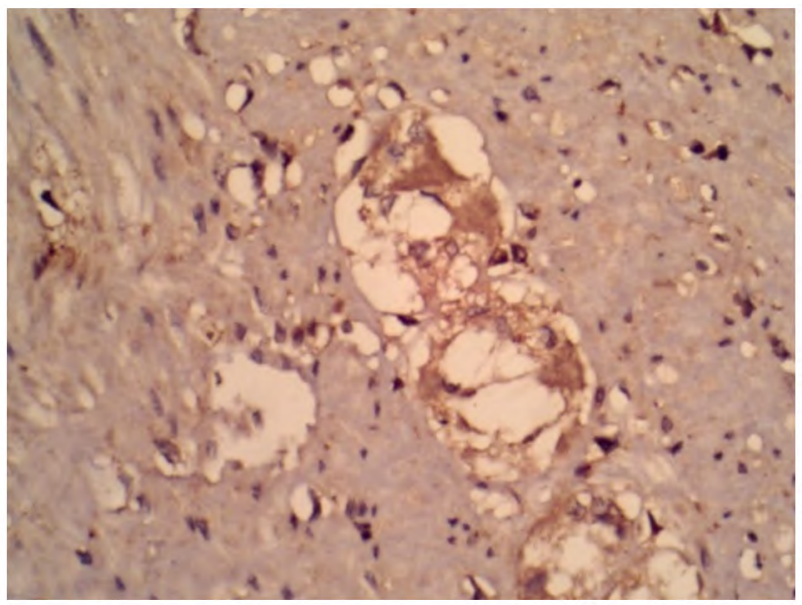

(B)

Fig. (1): Positive internal controls for CASK antibody. (A) Basolateral localization of stain in normal mucosa. (B) Group of ganglion cells between the layers of colonic wall smooth muscle fibers showing cytoplasmic stain (original magnification $\mathrm{X} 400)$.

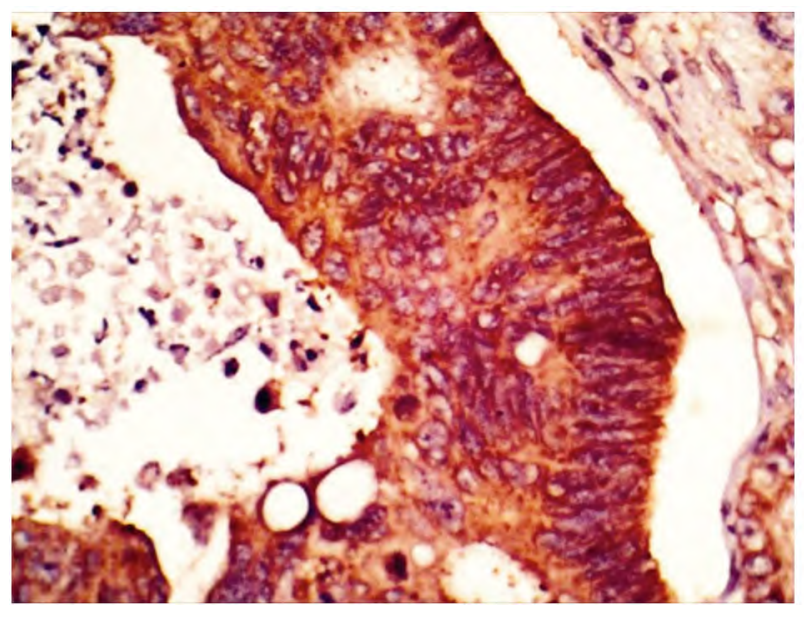

Fig. (2): Pure cytoplasmic pattern of expression of CASK in colon adenocarcinoma, $\mathrm{IS}=3$ (original magnification $\mathrm{X} 400)$.

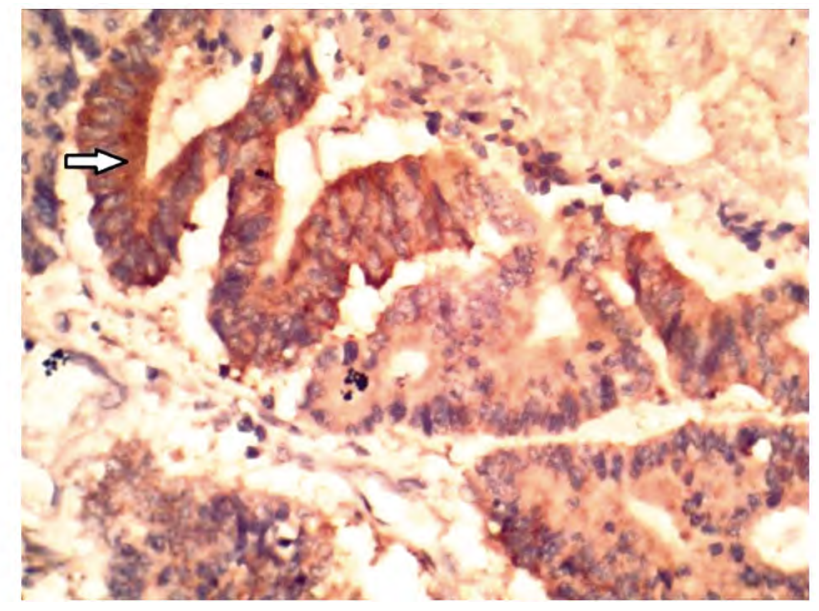

Fig. (3): Cytoplasmic distribution of CASK at moderate intensity (IS=2) with focal accentuation (arrow) (original magnification X400). 


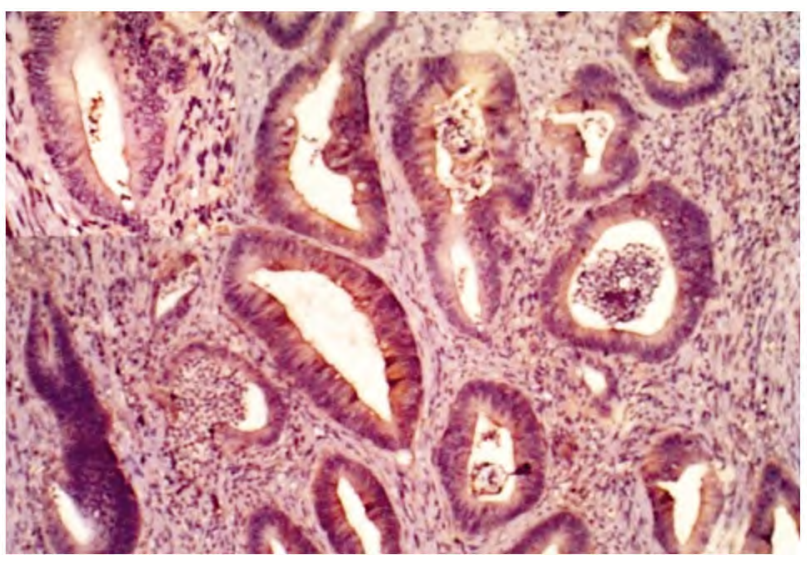

Fig. (4): Low grade colorectal carcinoma section negative for CASK (IS=1), with inset higher magnification (X400) of a mild focal cytoplasmic stain (original magnification X100).

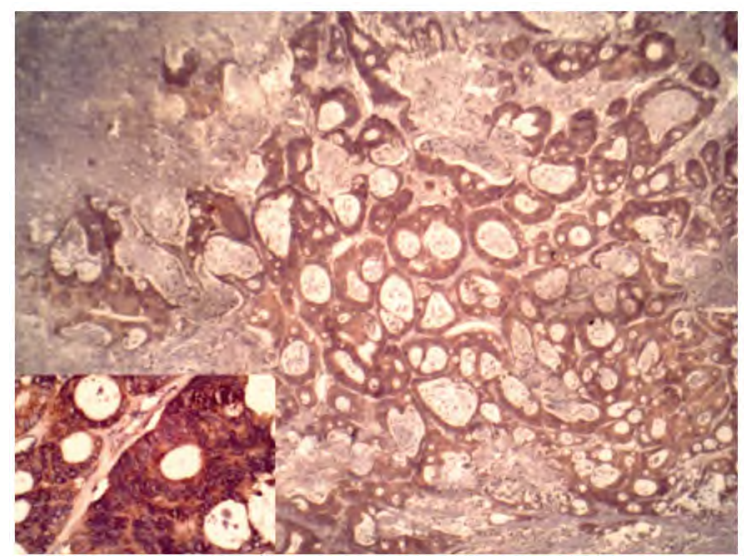

Fig. (6): Strong homogenous staining for CASK (IS=3) in low grade colorectal cancer cells. Inset higher magnification (X400) showing pure cytoplasmic pattern (original magnification X40).

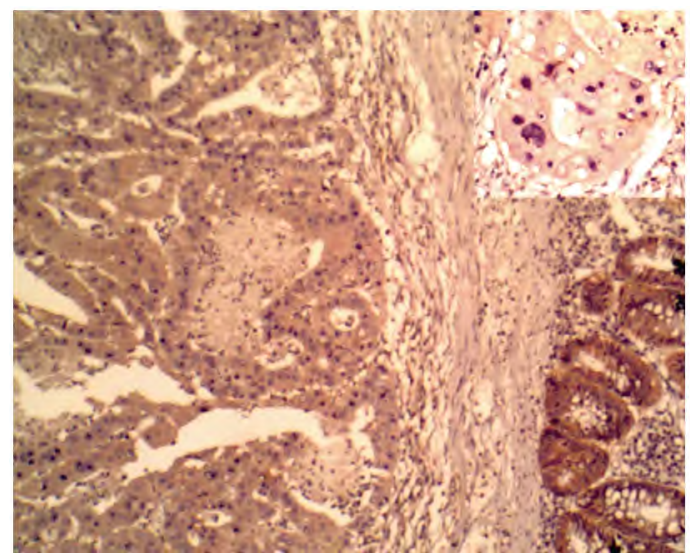

Fig. (8): High grade colon adenocarcinoma with mild cytoplasmic stain (-ve), showing positive staining of normal glands on the right (internal control. Note the high nuclear atypia at high magnification (Inset) (original magnification X100).

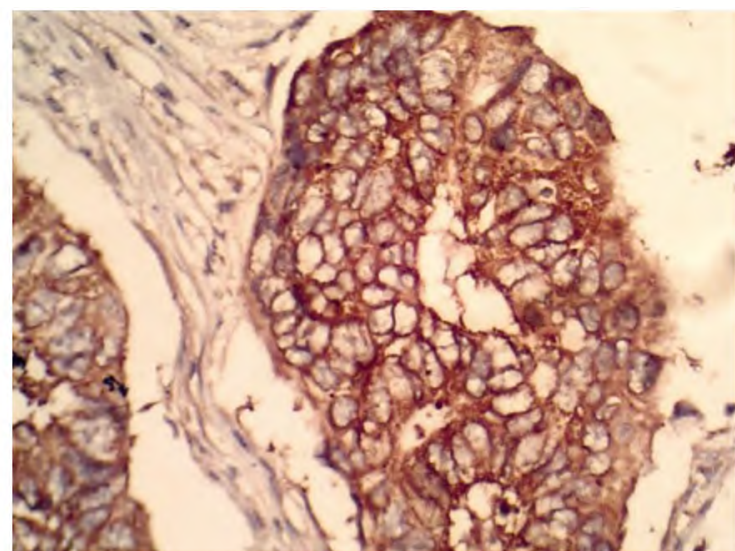

Fig. (5): Membranous pattern of staining of colon cancer with clear delineation of the cytomembranes, IS $=2$ (original magnification X400).

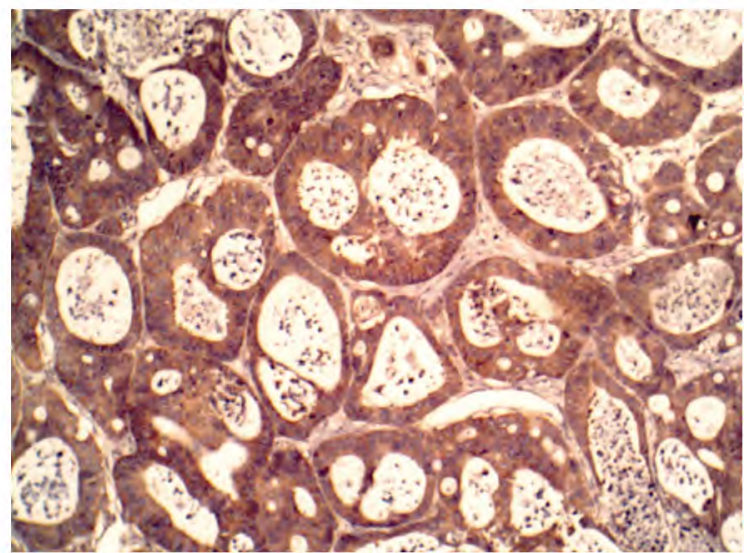

Fig. (7): Higher magnification of low grade CRC formed of variable sized and shaped acini, IS=3 (original magnification X100)

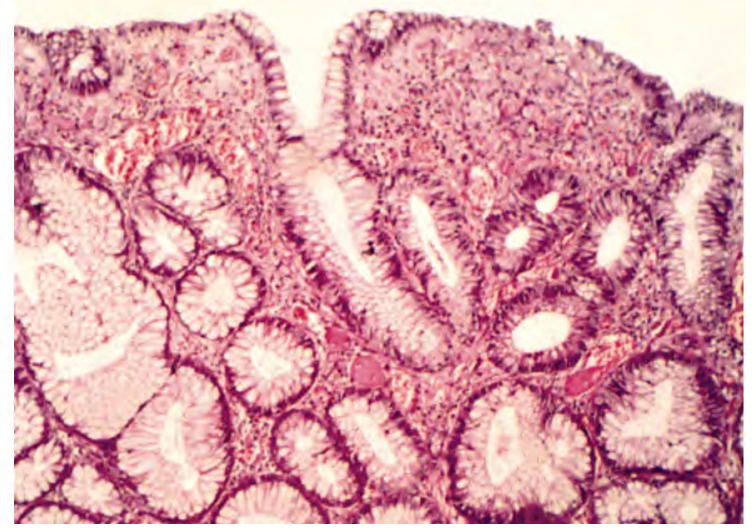

Fig. (9): Tubular adenoma with low grade dysplasia in $\mathrm{H}$ \& E stained section (original magnification X100). 


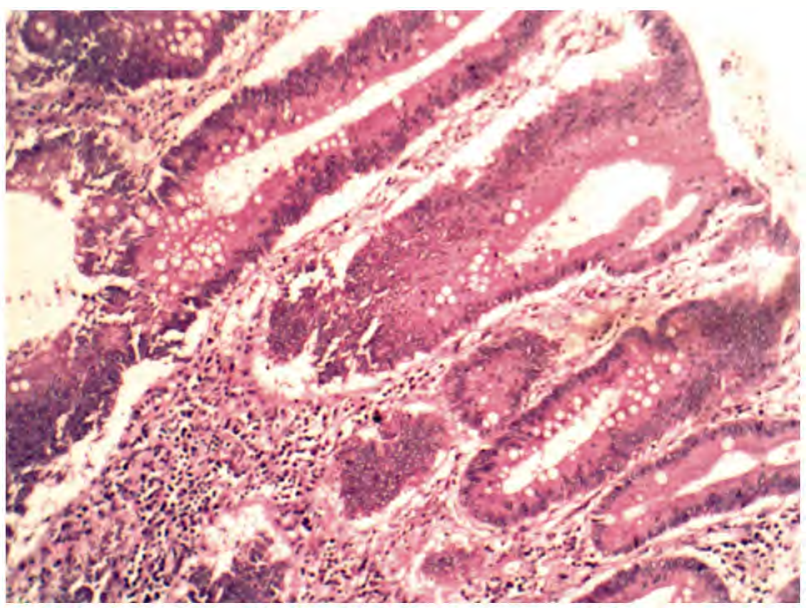

Fig. (10): Tubulovillous adenoma with high grade dysplasia showing complex architecture and nuclear atypia (original magnification X100).

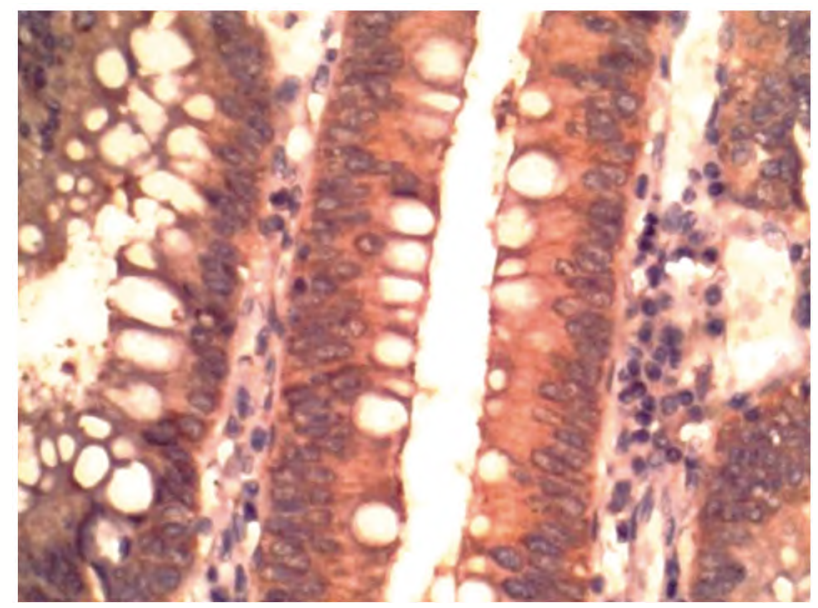

Fig. (11): Moderate intensity staining (IS=2) with CASK in a tubular adenoma with low grade dysplasia (original magnification X400).

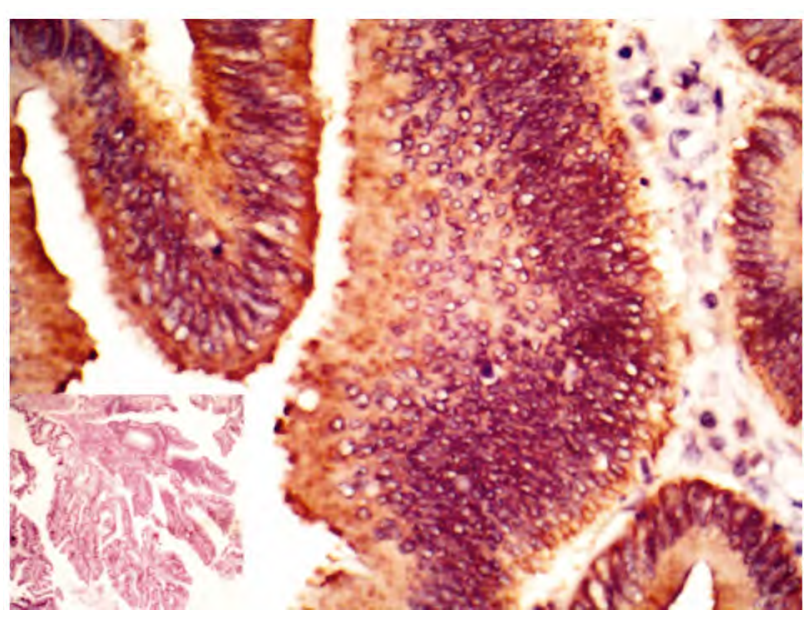

Fig. (12): Moderate intensity staining (IS=2) with CASK in a tubulovillous denoma (Inset) with high grade dysplasia (original magnification X400).
Table (1): Pattern of staining (PS) for CASK protein in CRC and adenoma cases.

\begin{tabular}{|c|c|c|c|c|c|c|}
\hline \multirow{2}{*}{$\begin{array}{l}\text { CASK } \\
\text { expression }\end{array}$} & \multicolumn{2}{|c|}{ Membranous stain } & \multirow{2}{*}{ Total } & \multicolumn{2}{|c|}{ Cytoplasmic stain } & \multirow{2}{*}{ Total } \\
\hline & Negativ & Positive & & Negativ & Positive & \\
\hline \multicolumn{7}{|l|}{$C R C N=42:$} \\
\hline Count & 19 & 23 & 42 & 4 & 38 & 42 \\
\hline$\%$ of total & $45.2 \%$ & $* 54.8 \%$ & $100 \%$ & $9.5 \%$ & $90.5 \%$ & $100 \%$ \\
\hline \multicolumn{7}{|l|}{ Adenomas } \\
\hline Count & 9 & 6 & 15 & 1 & 14 & 15 \\
\hline$\%$ of total & $60.0 \%$ & $40.0 \%$ & $100 \%$ & $6.7 \%$ & $93.3 \%$ & $100 \%$ \\
\hline
\end{tabular}

Table (2): Association of CASK protein expression with clinicopathological features of $\mathrm{CRC}$ cases. $\mathrm{N}=42$.

\begin{tabular}{|c|c|c|c|}
\hline \multirow{2}{*}{$\begin{array}{l}\text { Clinicopathological } \\
\text { variant }\end{array}$} & \multicolumn{2}{|c|}{ CASK expression } & \multirow{2}{*}{$p$-value } \\
\hline & Positive & Negative & \\
\hline \multicolumn{4}{|l|}{ TNM categories: } \\
\hline $\mathrm{I} / \mathrm{II}$ & $0(0 \%)$ & $15(35.7 \%)$ & \multirow[t]{2}{*}{$* 0.05$} \\
\hline III/IV & $6(14.3 \%)$ & $21(50.0 \%)$ & \\
\hline \multicolumn{4}{|l|}{ Grades: } \\
\hline Low grade & $1(2.4 \%)$ & $24(57.1 \%)$ & \multirow[t]{2}{*}{$* 0.001$} \\
\hline High grade & $5(11.9 \%)$ & $12(28.6 \%)$ & \\
\hline \multicolumn{4}{|c|}{ Lymphovascular invasion: } \\
\hline No & $23(54.8 \%)$ & $3(7.1 \%)$ & \multirow[t]{2}{*}{0.528} \\
\hline Present & $13(31.0 \%)$ & $3(7.1 \%)$ & \\
\hline
\end{tabular}

Table (3): Association between degree of dysplasia and CASK protein expression in adenomas. $\mathrm{N}=15$.

\begin{tabular}{llll}
\hline \multirow{2}{*}{ Dysplasia } & \multicolumn{2}{c}{ CASK expression } & p-value \\
\cline { 2 - 3 } & Negative & Positive & \\
\hline $\begin{array}{l}\text { Low grade: } \\
\text { Count } \\
\% \text { of total }\end{array}$ & 6 & 2 & 0.062 \\
$\begin{array}{l}\text { High grade: } \\
\text { Count } \\
\% \text { of total }\end{array}$ & $40.0 \%$ & $13.3 \%$ & \\
\hline
\end{tabular}

\section{Discussion}

Colorectal cancer is one of the leading causes of cancer related morbidity and mortality worldwide. It accounts for more than $9 \%$ of all cancer incidence. It is the third most common cancer globally and the fourth leading cause of death [2] Research suggests that the process of colorectal carcinogenesis usually follows a succession of histologic changes called the adenoma-to-carcinoma sequence. This sequence involves the accumulation of a combination of abnormalities in the genome allowing uncontrolled proliferation, incapacity for apoptosis and ultimately invasive and metastatic propensity [8].

The aim of our study was to evaluate the expression of Calcium/calmodulin-dependent serine 
protein kinase (CASK) in non-mucinous colorectal adenocarcinoma and its relation with clinicopathological variables of the disease (such as, tumor (T) stage, lymph node metastasis status, distant metastasis, lymphovascular invasion and grade of differentiation) with IHC staining.

The study was conducted at the Department of Pathology of Suez Canal University Teaching Hospital and included 42 non-mucinous CRC patients' samples and 15 adenomas biopsies.

CASK is expressed on the cell membranes of epithelial cells at different sites as in the choroid plexus epithelial cells, at synaptic junctions and hepatocytes. It was also found to be expressed in small and large intestinal epithelial cells; mainly at the baso-lateral regions of cell membranes and has been reported to mediate cell-cell adhesion and proliferation, which can contribute to tumorogenesis [5,9]. Our results showed that CASK protein was significantly overexpressed $(p<0.05)$ in CRC compared with normal samples. We therefore think that CASK overexpression might be a participant in the pathogenesis of CRC. We observed that the majority (52.8\%) of CRC samples showed both cytoplasmic and cytomembranous expression of CASK protein by immunohistochemistry, while only $20 \%$ of adenomas samples showed dual pattern of staining. This could suggest that besides CASK role in cell-cell interaction, its mobilization into the cytoplasmic pool might indicate it has a role in the control of cell growth and proliferation. Membranous staining pattern showed a statistically significant correlation with CASK protein overexpression $(p<0.05)$. This membranous pattern of staining could support our observation that CASK is a good prognostic marker. CASK protein localization on the cytomembrane might contribute to better tumor cell cohesion, preserved architecture and polarity.

Our results are accordant with a previous study that used western blot and IHC technique to measure the expression of the CASK protein and its gene in $156 \mathrm{CRC}$ tissue samples and compared it with its expression in 42 of normal and adenoma samples. Seventy three percent of their CRC samples displayed high expression while $84.2 \%$ of adenoma samples displayed low expression. Their results indicated that CASK was up-regulated in CRC compared with the other non-malignant tissue types $[4,10,11]$.

We also demonstrated for the first time significantly high levels of expression within adenomas with high grade dysplasia. Such results are consist- ent with the adenoma-carcinoma model of CRC progression $[6,12]$ and could indicate that CASK is involved along with other biomarkers in the progression of benign adenomatous polyps into highly dysplastic variant and then finally into invasive carcinomas.

CASK overexpression was significantly correlated with both TNM stage and grade of differentiation $(p<0.05)$. We observed significantly higher expression in tumor samples with early stages (I/II) rather than advanced stage (III/IV) and with low grade tumors rather than high grade ones.

We suggest that CASK protein might be a marker of well-differentiated, less aggressive tumors and its overexpression might be suggestive of favorable prognosis. CASK protein mainly functions as cell-cell adhesion molecule and is involved in regulation of epithelial cell polarity [7]. This being said, CASK overexpression might contribute to better tumor cell-cell cohesion and preserved architecture of tumor cells and therefore lower tumor grade. Our results in this aspect are accordant with a previous study of CASK expression analysis in cell lines. They found that induced CASK overexpression, with the resulting increase in the level of CASK protein, lead to significant decrease in the rates of cell growth and proliferation, while inhibition of about $68 \%$ of endogenous CASK protein production resulted in significant increase of the cell growth rates. Together, from these results they concluded that CASK may play an important role in inhibiting cell growth and proliferation beside its role in cell-cell adhesion [8]. On the other hand, a previous study on CRC associated CASK overexpression with advanced tumor stage, unfavorable prognosis and poor survival [13]. This discrepancy might be due to the complexity of genetic factors involved in the pathogenesis of $\mathrm{CRC}$ and influencing its behavior. Different genetic profiles of populations of different ethnicities should be considered. The relatively small number of high grade CRC samples (17 cases) in our study might be a factor for limitation.

\section{References}

1- HAGGAR F.A. and BOUSHEY R.P.: "Colorectal Cancer Epidemiology: Incidence, Mortality, Survival, and Risk Factors". Clinics In Colon And Rectal Surgery, 22 (4), 2009.

2- VERUTTIPONG D., SOLIMAN A.S., GILBERT S.F., BLACHLEY T.S., et al.: "Age distribution, polyps and rectal cancer in the Egyptian population-based cancer registry". W.J.G., 18 (30): 7: 92-8, 2012.

3- IBRAHIM A.S., KHALED H.M., MIKHAIL N.N., BARAKA H. and KAMEL H.: "Cancer Incidence in Egypt: 
Results of the National Population-Based Cancer Registry Program". Journal of Cancer Epidemiology, 2014: 18: 341-58, 2014

4- WEI J.L., FU Z.X., FANG M., ZHOU Q.Y., ZHAO Q.N., et al.: "High expression of CASK correlates with progression and poor prognosis of colorectal cancer". Tumor Biol., 35: 10: 9185-96, 2014.

5- COHEN A.R., WOODS D.F., MARFATIA S.M., WALTHER Z., CHISHTI A.H. and ANDERSON J.M.: "Human CASK/LIN-2 Binds Syndecan-2 and Protein 4.1 and Localizes to the Basolateral Membrane of Epithelial Cells". The Journal of Cell Biology, 142 (1), 1998.

6- HARRISON S. and BENZIGER H.: "The molecular biology of colorectal carcinoma and its implications: A review". The Surgeon, 9 (4): 200-10, 2011.

7- MARTINEZ-ESTRADA O.M., V.A., BREVIARIO F., ORSENIGO F., DEJANA E. and BAZZONI G.: "Association of junctional adhesion molecule with calcium/ calmodulin-dependent serine protein kinase (cask/lin-2) in human epithelial caco-2 cells". J. Biol. Chem., 276: 9291-6, 2001.

8- B.V. NAINI and R.D. ODZE: "Advanced precancerous lesions (APL) in the colonic mucosa." Best Pract. Res. Clin. Gastroenterol., 27 (2): 235-56, 2013.
9- MARTINEZ-ESTRADA O.M., V.A., BREVIARIO F., ORSENIGO F., DEJANA E. and BAZZONI G.: "Association of junctional adhesion molecule with calcium/ calmodulin-dependent serine protein kinase (cask/lin-2) in human epithelial caco-2 cells". J. Biol. Chem., 276: 9291-6, 2001

10- LOEB L.A., L.K. and ANDERSON J.P.: "Multiple mutations and cancer." Proc. Natl. Acad. Sci. USA, 100: 77681, 2003.

11- PASCHOS K.A., C.D. and BIRD N.C.: "The role of cell adhesion molecules in the progression of colorectal cancer and the development of liver metastasis". Cell Signal, 21 (5): 665-74, 2009.

12- SANJAY HARRISON and HARRISON BENZIGER: "The molecular biology of colorectal carcinoma and its implications: A review". The Surgeon, Journal of the Royal Colleges of Surgeons of Edinburgh and Ireland, 9 (4): 200-10, 2011.

13- VICENTE C.M., RICCI R., NADER H.B. and TOMA L. "Syndecan-2 is upregulated in colorectal cancer cells through interactions with extracellular matrix produced by stromal fibroblasts". B.M.C. Cell Biology, 14: 25-38, 2013. 


\section{تمثيل إنزيم كاسك فى سرطان القولون والمستقييم الغير مخاطى

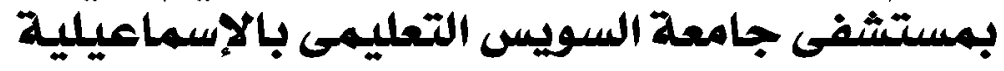

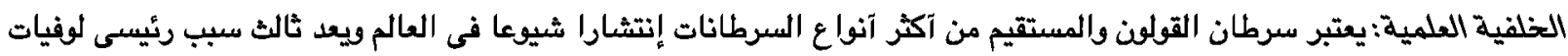

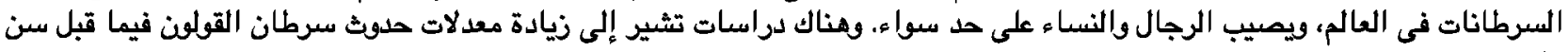

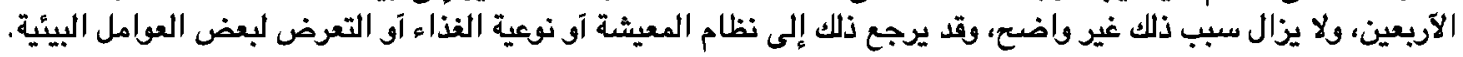

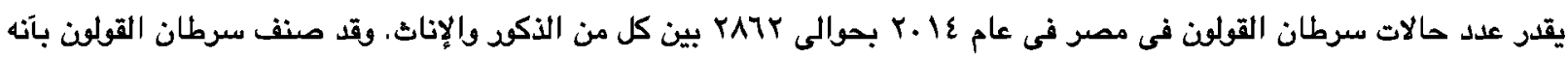

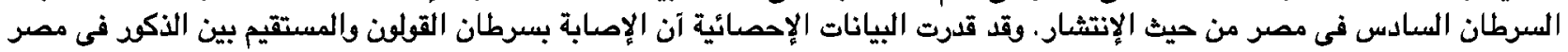

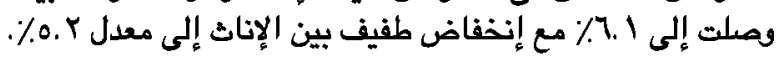

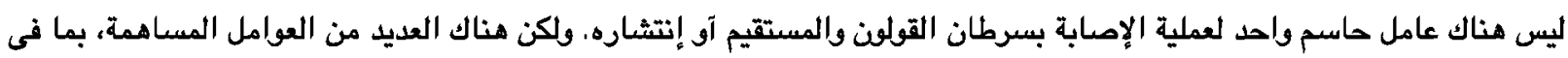

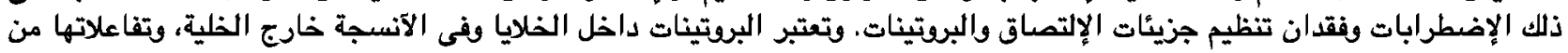

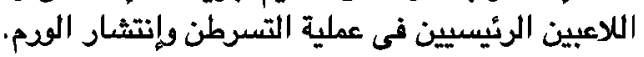

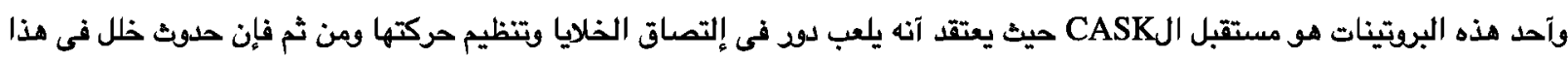

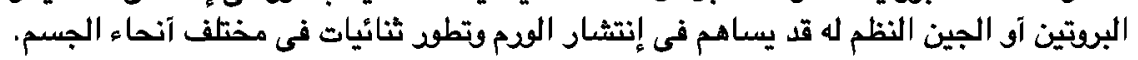

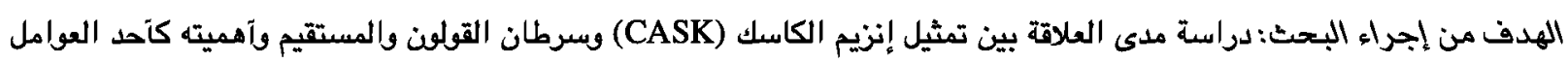
المنترة في تطود المرض. المراء.

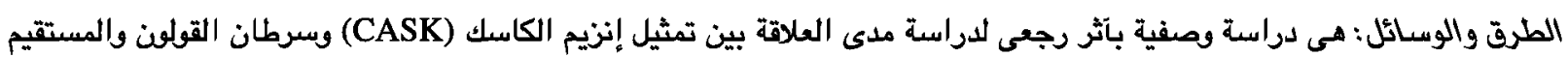

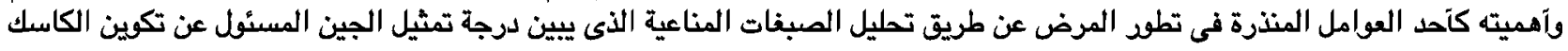
فى نسيج الودم.

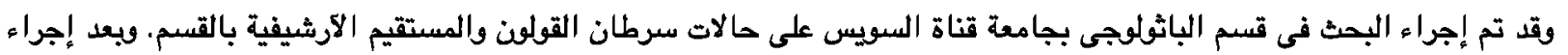

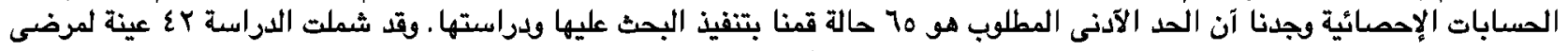

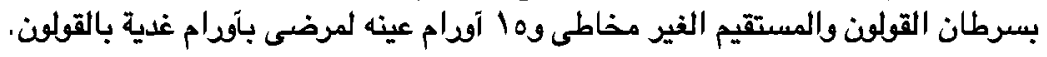
قمنا بجمع البيانات التالية عن المرضئ العمر والجنس ومكان الورم فى الآمعاء وحجمه، وكذلك المرحلة المرضية، درجة تميز الخلايا السرطانية.

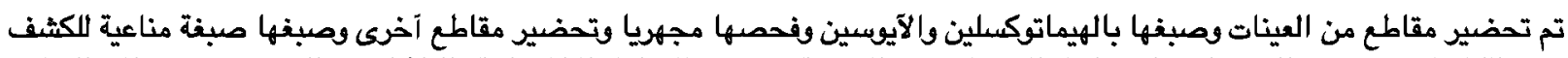

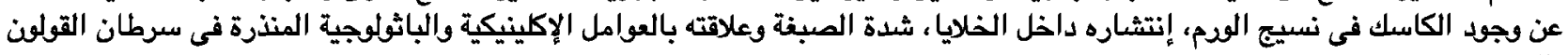
والمستقيم.

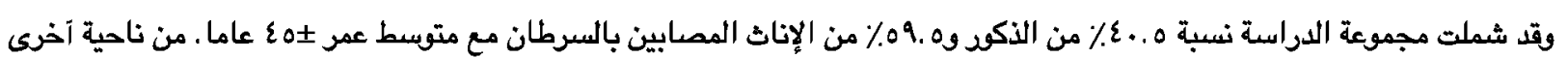

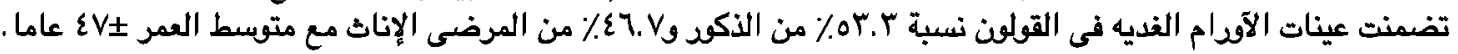

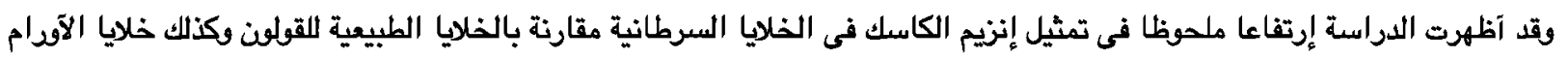

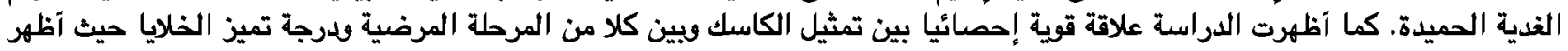

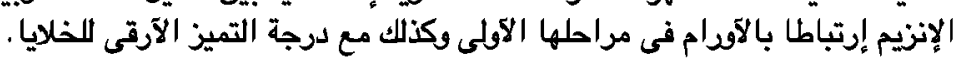

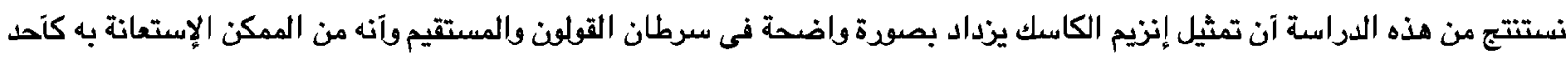
المؤشرات الإيجابية فى تطود المرض وإنتشئ إنريمارها 